\title{
BMJ Open Study protocol for a framework analysis using video review to identify latent safety threats: trauma resuscitation using in situ simulation team training (TRUST)
}

\author{
Mark Fan, ${ }^{1,2}$ Andrew Petrosoniak, ${ }^{3,4}$ Sonia Pinkney, ${ }^{2}$ Christopher Hicks, ${ }^{3,4}$ \\ Kari White, ${ }^{5}$ Ana Paula Siquiera Silva Almeida, ${ }^{6}$ Douglas Campbell, ${ }^{5,7}$ \\ Melissa McGowan, ${ }^{3}$ Alice Gray, ${ }^{3}$ Patricia Trbovich ${ }^{1,2,8}$
}

To cite: Fan M,

Petrosoniak A, Pinkney S, et al. Study protocol for a framework analysis using video review to identify latent safety threats: trauma resuscitation using in situ simulation team training (TRUST). BMJ Open 2016;6 e013683. doi:10.1136/ bmjopen-2016-013683

- Prepublication history and additional material is available. To view please visit the journal (http://dx.doi.org/ 10.1136/bmjopen-2016013683)

Received 2 August 2016 Accepted 13 October 2016

CrossMark

For numbered affiliations see end of article.

Correspondence to

Mark Fan;

mark.fan@nygh.on.ca

\section{ABSTRACT}

Introduction: Errors in trauma resuscitation are common and have been attributed to breakdowns in the coordination of system elements (eg, tools/ technology, physical environment and layout, individual skills/knowledge, team interaction). These breakdowns are triggered by unique circumstances and may go unrecognised by trauma team members or hospital administrators; they can be described as latent safety threats (LSTs). Retrospective approaches to identifying LSTs (ie, after they occur) are likely to be incomplete and prone to bias. To date, prospective studies have not used video review as the primary mechanism to identify any and all LSTs in trauma resuscitation.

Methods and analysis: A series of 12 unannounced in situ simulations (ISS) will be conducted to prospectively identify LSTs at a level 1 Canadian trauma centre (over 800 dedicated trauma team activations annually). 4 scenarios have already been designed as part of this protocol based on 5 recurring themes found in the hospital's mortality and morbidity process. The actual trauma team will be activated to participate in the study. Each simulation will be audio/video recorded from 4 different camera angles and transcribed to conduct a framework analysis. Video reviewers will code the videos deductively based on a priori themes of LSTs identified from the literature, and/or inductively based on the events occurring in the simulation. LSTs will be prioritised to target interventions in future work.

Ethics and dissemination: Institutional research ethics approval has been acquired (SMH REB \#15-046). Results will be published in peer-reviewed journals and presented at relevant conferences. Findings will also be presented to key institutional stakeholders to inform mitigation strategies for improved patient safety.

\section{INTRODUCTION}

Error is ubiquitous in trauma resuscitation and despite significant advances in trauma

\section{Strengths and limitations of this study}

Latent safety threats (LSTs) are defined as 'system-based threats to patient safety that can materialize at any time and are previously unrecognized by healthcare providers, unit directors or hospital administration'.

- This protocol uses a novel video-based framework analysis (FA) as the primary means of identifying LSTs during in situ trauma simulations.

- The FA, applied by human factors experts, provides a matrix output that facilitates interprofessional review of the data, showing patterns of LSTs within and between simulations, and provides a high degree of transparency and traceability in how LSTs are identified, interpreted and coded.

- Our study is limited to 12 simulations at a single trauma centre and it is not designed to capture the true incidence of LSTs possible in actual trauma resuscitations; additionally, participants may not perform as they would in actual trauma resuscitations (eg, the Hawthorne effect).

care,${ }^{1-4}$ medical error remains a preventable cause of adverse events and serious harm. ${ }^{5}$ The unpredictable nature of trauma care, combined with inherent diagnostic uncertainty, variable patient numbers (ie, single vs multiple injured) and high patient acuity, challenges even the most experienced teams. ${ }^{6}$ Errors in trauma care are often the result of a departure from best practices and/or errors in judgement that manifest from a series of events that coalesce to decrease patient safety. ${ }^{1}{ }^{3} \quad 6-8$ The overarching goal of the proposed study is to identify latent safety threats (LSTs) in trauma, defined as 'system-based threats to patient safety that can materialize at any time and 
are previously unrecognized by healthcare providers, unit directors or hospital administration'. ${ }^{\prime}$

\section{Retrospective versus prospective studies in trauma resuscitation}

Multiple retrospective studies in trauma care have established that errors result in preventable patient deaths. ${ }^{13467}$ Specifically, delays in diagnosis and management, errors in clinical judgement, technical errors (eg, failure to insert catheter) and procedural errors (eg, failure to perform a step in a standardised protocol) are all frequent causes of preventable deaths in trauma.

While retrospective analyses provide important information to direct investigations of prior adverse events, it is equally, if not more, important to prospectively identify potential risks to build resiliency in the trauma system. ${ }^{10}$ Retrospective studies often rely on chart review and/or staff recall (eg, morbidity and mortality reviews), yet the inherent limitations associated with this reflective approach fail to capture the complexity that lead to error. ${ }^{11}$ In contrast, prospective studies can be designed to comprehensively record all activities taking place during a trauma resuscitation followed by a thorough analysis of how specific activities or equipment converge to produce errors. Here, we propose a prospective study designed to identify LSTs.

\section{Prospective study design for LST identification in trauma resuscitation}

Human factors (HF) and ergonomics has been used increasingly to study errors in healthcare ${ }^{12} \mathrm{HF}$ is the scientific discipline concerned with the understanding of interactions among humans and other elements of a system $^{13}$ and it focuses on optimising system design based on the strengths and limitations of human performance. An HF perspective attempts to account for the needs of all users based on the interactions between multiple system elements, ${ }^{14-20}$ including but not limited to: (1) individual skills/knowledge and team interaction (eg, trauma team possesses sufficient knowledge and skill to stabilise the patient), (2) tools/technology (eg, equipment availability, usability and/or malfunctions), (3) tasks (eg, simple vs complex, routine vs unanticipated), (4) physical environment and layout (eg, space around the patient's bed) and (5) organisational characteristics (eg, reliability of processes that coordinate delivery of blood products to the trauma bay). By evaluating the potential for mismatches between these elements, the HF perspective offers a starting point for LST identification.

Healthcare simulation offers a valuable approach to evaluate teams and processes and identify LSTs without harm to patients. Prospective studies in emergency medicine and other fields in critical care medicine (neonatal intensive care, paediatric intensive care, obstetrics, paediatric emergency medicine) have used simulation laboratories and in situ simulation (ISS) for LST identification. ${ }^{9}{ }^{21-29}$ ISS or 'simulation that is physically integrated into the clinical environment ${ }^{30}$ is particularly effective for LST detection. ${ }^{25} 263132$ In fact, for this purpose, ISS has been shown to be superior when compared with simulation laboratories. ${ }^{9} 2233$ The real-life applicability of ISS allows simulation facilitators to recreate previously reported critical events in the exact working environment in which they originally occurred, providing a unique opportunity to explore and learn from past challenges. In addition, simulation in the actual workplace can reduce the cost and logistical barriers associated with traditional laboratory-based, interprofessional simulation sessions ${ }^{34}{ }^{35}$ (eg, clinical environment and equipment already setup by default, reduced travel time for participants). In sum, ISS offers a resource-efficient and highly effective strategy to prospectively identify LSTs.

To the best of our knowledge, there are no prospective studies in an adult trauma centre that use a rigorous review of ISS video recordings as the primary means to identify any and all LSTs during trauma resuscitation. Instead, video review is used to verify or clarify LSTs identified by other mechanisms such as postsimulation debriefings and questionnaires. ${ }^{9}$ Video recordings, however, have been used to review real trauma cases for LSTs. ${ }^{36-40}$ For example, video review provided important observations related to communication failures among trauma teams. ${ }^{41}$ We consider video review during trauma ISS an underutilised but essential tool to identify any and all LSTs because it allows for detailed review and consideration of all HF elements. Video review serves to minimise recall bias inherent with traditional, non-video recorded mortality and morbidity rounds and other retrospective analyses. The superiority of video recordings compared with self-reporting tools and chart review have been previously studied, revealing a greater number of 'performance deficiencies' and Advanced Trauma Life Support protocol deviations than other methods. ${ }^{36} 42$ Video review also has greater value for incident analysis than direct observation. ${ }^{43}$ The dynamic environment and complex interactions of trauma care necessitate comprehensive methods to capture safety threats otherwise unnoticed by the preoccupied trauma team. This supports the importance of prospectively collected video recordings during carefully designed ISS scenarios.

Previous ISS studies have typically used a deductive approach to identify LSTs. Deductive approaches allow researchers to preselect categories of LSTs based on past literature and theories. ${ }^{44}$ For example, a past study using ISS in trauma resuscitation has categorised LSTs by themes such as medications, equipment and physical environment, team communication and hospital systems. ${ }^{9}$ However, the use of pre-existing themes to organise data collection and analysis may unduly bias against unanticipated LSTs that have not been previously discussed or identified. Unanticipated LSTs may be better identified using an inductive approach, whereby 
the researcher generates themes directly from the data by freely coding observations without restrictions. These themes can be iteratively refined with subsequent reflection and integration of new and/or related LSTs. In this study, we use a combined approach (ie, deductive and inductive) to explore specific themes of LSTs recognised in HF literature while allowing for the flexibility of uncovering other themes based on our observations. ${ }^{45}$

\section{Framework analysis for ISS video recordings}

We propose the use of framework analysis (FA) ${ }^{44}$ to analyse video recordings from ISSs. FA has been used in several settings, including social policy research, midwifery, nursing and health psychology. ${ }^{46}$ FA has several benefits: ${ }^{44}$

- it allows for a combination of deductive and inductive analyses;

- it works well within multidisciplinary health research comprised of multiple team members;

- it supports transparency and greater engagement with the full study team;

- it assists in managing large data sets where obtaining a holistic, descriptive overview of the entire data set is desirable, in part due to its matrix output.

A more thorough discussion of the FA is included in A modified framework analysis to identify LSTs section.

\section{Summary}

The primary goal of the present study is to apply an HF perspective to the identification of LSTs in trauma care using ISS with video review as the primary data collection strategy. Past studies typically used postsimulation debriefs, questionnaires, surveys and/or live observations to identify LSTs; these are secondary strategies used in our study. To the best of our knowledge, this is the first study to apply an FA using risk-informed in situ trauma simulation scenarios to identify LSTs. Our approach may capture more LSTs than previous study designs and therefore provide a more comprehensive view of the aetiology of errors during trauma care within a mature trauma centre.

\section{METHODS AND ANALYSIS}

The overall study design is presented in figure 1. The trauma registry case review began in July 2015 . We anticipate completion of the study by November 2016 .

\section{Objective}

To identify and prioritise LSTs using unannounced interprofessional in situ trauma simulation sessions that recur on a regular basis. LST detection will occur via two processes:

1. video-review by HF specialists with expertise in LST identification, and

2. a focused debrief of trauma team members (verbal and written).

\section{Setting}

The proposed study will occur at St Michael's Hospital, a Level 1 trauma centre in Toronto, Canada, with 75000

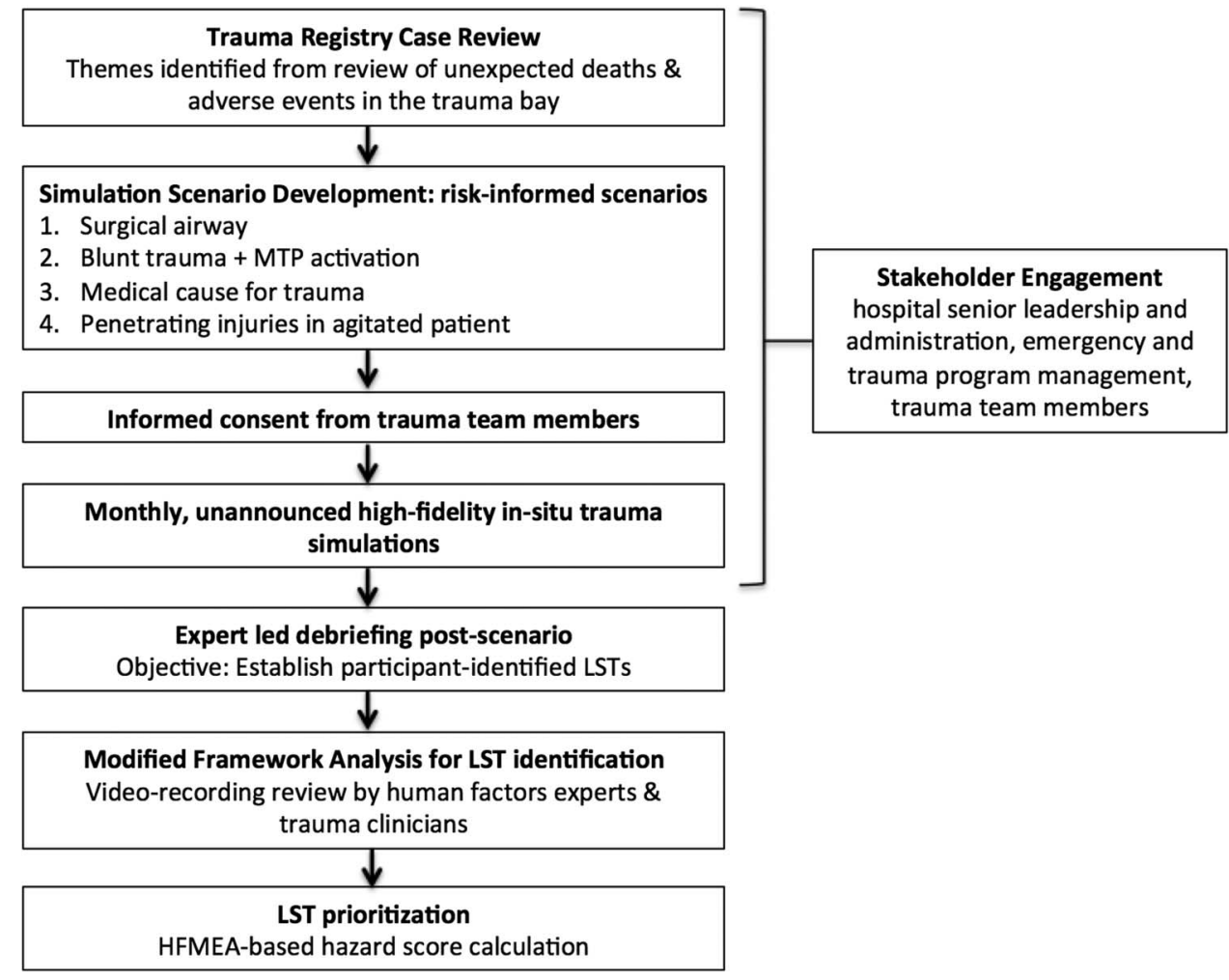

Figure 1 Study design. LST, latent safety threats; HFMEA, healthcare failure mode and effect analysis. 
emergency department (ED) visits annually including 800 ( $\sim 33 \%$ with injury severity score $>16)$ trauma team activations managed by the institution's dedicated on-call trauma team. The trauma team is activated by the ED triage nurse through the hospital paging system prior to arrival of a critically injured patient. Less commonly, a trauma team activation occurs after the patient has arrived in the ED at the discretion of the triage nurse or emergency physician. In this study, the trauma team will be notified through the established activation process with no mention of 'simulation' to preserve the fidelity of participant response times.

\section{Participants}

Study participants will include all members of the on-call trauma team on the day of the simulation. Trauma team members rotate daily; as such, there is rarely an identical team composition. The trauma team is led by a staff physician in emergency medicine or general surgery who has no additional clinical responsibilities during their on-call shift. The team consists of two ED nurses who are staffed elsewhere in the ED prior to the patient's arrival, two general surgery residents, one to two orthopaedic residents, an anaesthesia resident, a respiratory therapist, a social worker, an X-ray technician and a clinical assistant. An on-call staff trauma surgeon will attend at the trauma team leader's discretion or if the patient requires operative management.

The consent process will occur 1-2 months in advance of the start of the study and will include all providers who may be involved in the care of a trauma patient within the ED (see the Participants and consent section for additional details). With the exception of the on-call trauma team leader, participants will not be notified of time and date of the ISS. On arrival to the trauma bay, all team members will be given the opportunity to decline participation regardless of their previous consent status. To most accurately observe participant task prioritisation, there will be no supplemental staffing provided during the ISS; however, all participating staff will have the opportunity to depart at any time for urgent clinical responsibilities. Research ethics board approval was acquired for this study (see the Participants and consent section).

\section{Scenarios}

\section{Scenario design}

As part of the preparation for this protocol, scenarios have already been designed. A retrospective chart review of previous patient cases where adverse events or unexpected deaths were identified was performed. Inclusion criteria included patients who activated a trauma code at St Michael's Hospital between 1 January 2013 and 31 December 2014. During this time, 1473 trauma team activations occurred and 132 cases $(9.0 \%)$ were identified through our institution's morbidity and mortality process. Twenty-six cases (26/132,
$19.7 \%$ ) were subject to a more detailed review due to low probability of death or deviations from protocol adherence. Two trauma physicians reviewed these 26 cases in depth with expertise in simulation (AP and $\mathrm{CH})$. Five broad themes were identified and reviewed with the chief of trauma surgery and the trauma coordinator responsible for the trauma database to confirm theme relevance. The following themes were selected for scenario inclusion: difficult airway, massive transfusion protocol activation, medical causes for trauma, penetrating injury mechanisms and agitated/uncooperative patients. Simulation scenarios for the study were risk-informed, derived from actual cases, using key clinical and system elements related to each theme. Additionally, the scenarios were designed such that the HF elements most prone to LSTs varied. Table 1 describes each scenario and the mapping to HF elements where LSTs were considered likely. It is expected that each scenario will be executed a minimum of two times, and a maximum of five.

All four scenarios were sufficiently modified such that participants could not attribute them to the specific cases from which they were inspired. Each scenario is designed so the simulations can be conducted within $15 \mathrm{~min}$ to minimise disruption to actual clinical operations. All scenarios were pilot tested for clarity, flow and logistical considerations by a multidisciplinary team (ie, not study participants). Scenarios were modified based on pilot testing feedback and study team observations.

As suggested by Chiniara et $a l,{ }^{47}$ we selected scenarios that are optimised for simulation by ensuring patient acuity is high, and the 'opportunity' for the scenario's occurrence is low to medium.

\section{Functional task alignment of ISS}

We will design and conduct each session such that each simulation scenario meets the trauma team's clinical task demands, a concept referred to as functional task alignment. ${ }^{48}$ Instead of solely focusing on the physical resemblance the simulator has to a real patient, the functional properties of the ISS will be aligned with the predetermined objectives for the session. To this end, our simulations will occur with either a manikin or a standardised patient (SP) based on the functional task alignment required by the objectives. For example, an SP will be used when a key feature of the scenario involves interacting with and managing an uncooperative and agitated patient. Most important for our observation of LSTs realistic to trauma resuscitation, participants will have access to all the usual hospital resources (eg, equipment, activation of hospital procedures/processes). Additionally, participants will not know when simulations are scheduled (with the exception of the trauma team leader, as described in the Participants section); this is a key advantage to meet the functional properties that ISS provides compared with centre-based simulations. 
Table 1 Scenario characteristics and targeted human factors elements for latent safety threat identification

\begin{tabular}{|c|c|c|c|c|}
\hline Theme & Scenario description & $\begin{array}{l}\text { Scenario } \\
\text { characteristics }\end{array}$ & $\begin{array}{l}\text { Human factors } \\
\text { elements prone to } \\
\text { LSTs }\end{array}$ & Human factors rationale \\
\hline Surgical airway & $\begin{array}{l}\text { A patient arrives with considerable facial trauma } \\
\text { requiring immediate airway management. The } \\
\text { scenario is designed such that all techniques to } \\
\text { manage the patient's airway are ineffective } \\
\text { except for a surgical airway (eg, } \\
\text { cricothyroidotomy) }\end{array}$ & $\begin{array}{l}\text { Low opportunity } \\
\text { High acuity } \\
\text { Manikin }\end{array}$ & $\begin{array}{l}\text { Individual skill/ } \\
\text { knowledge and team } \\
\text { Interaction }\end{array}$ & $\begin{array}{l}\text { Trauma team members must escalate to surgical } \\
\text { airway (an infrequent occurrence) without } \\
\text { unnecessary delay. Cognitive biases against this } \\
\text { course of action must be overcome individually } \\
\text { and collectively } \\
\text { Infrequently used equipment must be collected } \\
\text { and used appropriately }\end{array}$ \\
\hline $\begin{array}{l}\text { Blunt trauma requiring } \\
\text { activation of the mass } \\
\text { transfusion protocol } \\
\text { (MTP) }\end{array}$ & $\begin{array}{l}\text { A patient arrives with an open book pelvic } \\
\text { fracture (and a misplaced pelvic binder) who } \\
\text { becomes haemodynamically unstable, requiring } \\
\text { a rapid administration of blood products among } \\
\text { other interventions }\end{array}$ & $\begin{array}{l}\text { Medium } \\
\text { opportunity } \\
\text { High acuity } \\
\text { Manikin }\end{array}$ & $\begin{array}{l}\text { Physical } \\
\text { environment and } \\
\text { layout } \\
\text { Organisational } \\
\text { characteristics }\end{array}$ & $\begin{array}{l}\text { The MTP process requires some staff to } \\
\text { physically navigate the hospital to deliver } \\
\text { paperwork and retrieve blood products } \\
\text { Interdepartmental coordination is required to } \\
\text { execute a mass transfusion protocol }\end{array}$ \\
\hline $\begin{array}{l}\text { Medical cause for } \\
\text { trauma }\end{array}$ & $\begin{array}{l}\text { A patient arrives after falling down a flight of } \\
\text { stairs due to a syncopal event. The patient } \\
\text { suffers a cardiac arrest on arrival requiring the } \\
\text { team to consider underlying medical conditions } \\
\text { in addition to critical injuries }\end{array}$ & $\begin{array}{l}\text { Medium } \\
\text { opportunity } \\
\text { High acuity } \\
\text { Manikin }\end{array}$ & $\begin{array}{l}\text { Individual skill/ } \\
\text { knowledge and team } \\
\text { interaction }\end{array}$ & $\begin{array}{l}\text { As individuals, and as a team, there is a need to } \\
\text { look beyond obvious blunt trauma and } \\
\text { haemorrhage considerations and consider } \\
\text { underlying medical causes }\end{array}$ \\
\hline \multirow[t]{3}{*}{$\begin{array}{l}\text { Penetrating injuries and } \\
\text { agitated patient }\end{array}$} & $\begin{array}{l}\text { A patient with penetrating stab wounds walks } \\
\text { into the ED, with a knife in the abdomen. The } \\
\text { patient is haemodynamically unstable and } \\
\text { requires expedient transfer to the operating } \\
\text { theatre for surgical intervention }\end{array}$ & $\begin{array}{l}\text { Medium-High } \\
\text { opportunity } \\
\text { High acuity } \\
\text { Standardised } \\
\text { patient }\end{array}$ & $\begin{array}{l}\text { Individual Skill/ } \\
\text { knowledge and team } \\
\text { interaction }\end{array}$ & $\begin{array}{l}\text { Trauma team members must rapidly manage } \\
\text { penetrating injuries that could rapidly lead to } \\
\text { destabilisation. Literature shows delays or } \\
\text { insufficient control of haemorrhage are a common } \\
\text { error in trauma resuscitation }{ }^{13467}\end{array}$ \\
\hline & & & $\begin{array}{l}\text { Organisational } \\
\text { characteristics }\end{array}$ & $\begin{array}{l}\text { Rapid mobilisation of operating theatre resources } \\
\text { for critically injured patients presents logistical } \\
\text { challenges related to resource allocation, patient } \\
\text { transport and interdepartmental communication }\end{array}$ \\
\hline & & & Task & $\begin{array}{l}\text { Task of treating an alert and agitated patient that } \\
\text { may actively resist or question treatment is } \\
\text { complex and also interferes with team } \\
\text { communication }\end{array}$ \\
\hline
\end{tabular}




\section{Materials/equipment}

Patient simulator/SP and equipment

To best evaluate our existing clinical equipment and team workflow, all equipment and systems used during the care of real trauma patients will be available during the study simulations. Simulation scenarios 1-3 (as described in table 1) will be performed using a SimMan 3G Human Patient Simulator (Laerdal Medical, Stavanger, Norway). Our institution's simulation centre team will be responsible for the set up of equipment, acquisition of video and maintenance and trouble-shooting of equipment-related issues. An actor will be recruited from the University of Toronto SP programme for scenario 4, the 'penetrating injuries' scenario.

\section{Video equipment and setup}

Four video cameras will be placed throughout the trauma bay (2 GoPro Hero4 2015, 1 GoPro Hero and 1 Sony Handycam Exmor R) for all simulation sessions. Two cameras will be positioned to provide a wide-angle perspective of the trauma bay and two cameras will be positioned above the patient to capture clinical care activities in more detail (figure 2).

\section{Audio recording setup}

Audio will be captured using an overhead microphone (Aputure V Mic D1 Directional Condenser Shotgun) hanging above the patient from the ceiling; handheld voice recorders (Sony IC Recorder ICD-UX533) will also be placed in the medication nursing area and phones to capture conversations farther from the bedside.

\section{Questionnaire}

We will administer a questionnaire as part of the postscenario debrief to solicit LSTs identified by participants related to the HF elements described earlier (individual skills/knowledge and team interaction; tools/technology; tasks; physical environment and layout; organisational characteristics). The questionnaire will be designed by the study team. See online supplement 1 for the questionnaire.

\section{Procedure for conducting simulation}

The procedure for conducting each ISS is as follows:

1. The study investigators will consult with the staff emergency physician and charge nurse in the ED for approval to conduct the simulation on the study day.

2. The simulation control centre will be established to operate the patient monitor and alter vital signs according to the clinical situation while responding to the participants' interventions (eg, respond to participants' interventions).

3. The research team (ie, simulation experts, research coordinators, clinical experts, HF experts) will attend each simulation to operate the simulator and the cameras, and provide technical/logistical support as needed.

4. Two members of the research team will act as confederates; one in the role of a respiratory therapist and the other as a paramedic providing a handover report to the trauma team. This is performed to ensure that the trauma team is oriented to the scenario and receives a report on the patient as per usual processes, as well as to address any limitations inherent in simulation (eg, participants may be unclear if

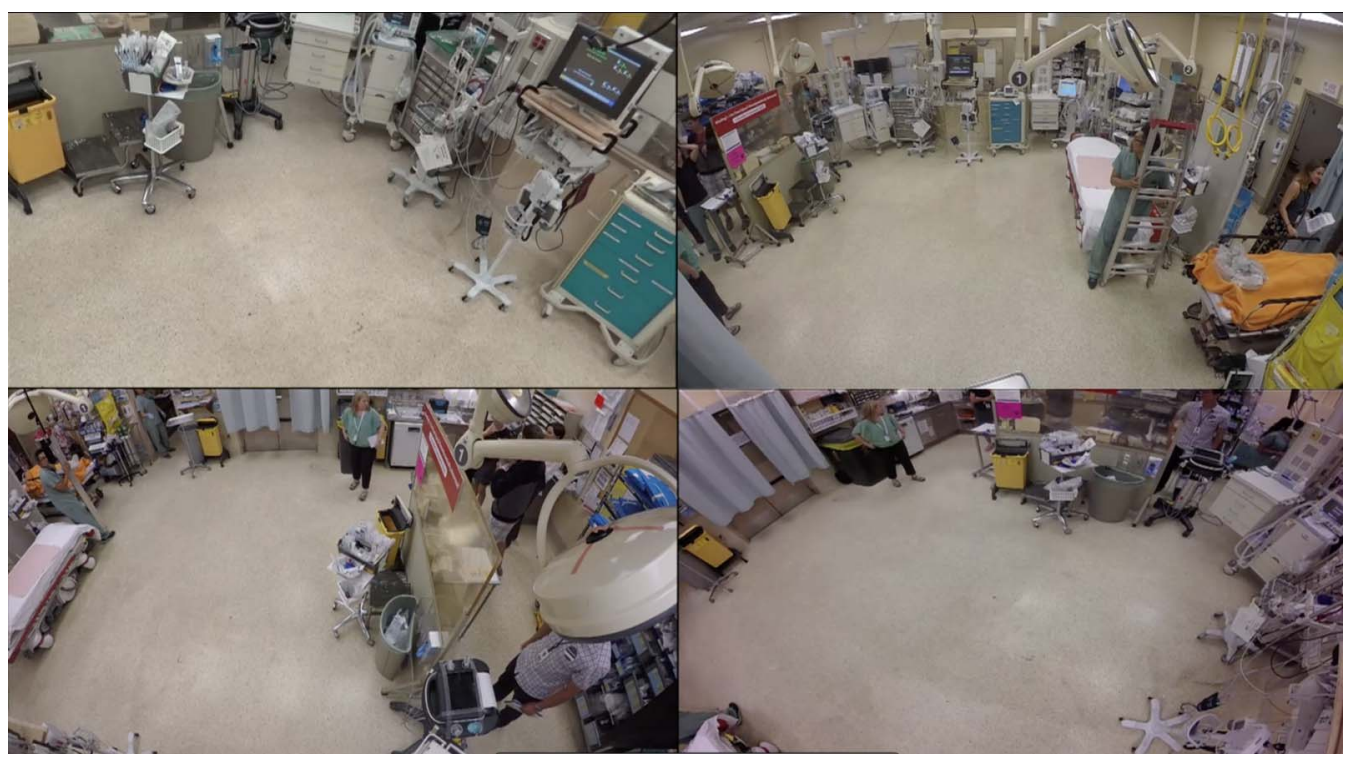

Figure 2 Top left is a focused view of patient care activities and the airway team; Top right focuses on a wide-angle view of the trauma bay (including X-ray vest area on the right); bottom left is a wide-angle view of the trauma bay from the patient's right side and offers a view of the entrances and exits; bottom right is a wide view of the patient care activities but also includes the medication nursing area behind the $\mathrm{X}$-ray wall. 
the manikin is capable of certain physiological indications and seek confirmation).

5. The trauma team will be notified in the usual manner via the established hospital paging system. The manikin or SP will be brought into the trauma bay $\sim 2$ min after the trauma team activation.

6. The trauma team will be instructed to care for the simulated patient in the same manner as an actual trauma patient. In the case of the SP, select members of the team may be informed about specific constraints related to the SP (see the Standardized patient section for further detail).

7. Based on published experiences of ISS, ${ }^{26} 49$ each scenario will run for $\sim 15 \mathrm{~min}$ followed by a $15 \mathrm{~min}$ semistructured debriefing (verbal and written questionnaire) led by two simulation and debriefing experts. The focus of the debriefing will be the identification of LSTs and follow three phases:

- First, the facilitator will inform all participants that the debrief will take no longer than $15 \mathrm{~min}$, that it will be confidential and that the purpose of the study is to identify barriers to safe and effective care rather than a focus on individual performance. Participants will be reminded to answer pagers or return to any acute patient care duties as necessary. The LST questionnaire will be handed to all participants at this time.

- Participants will then complete the written LST questionnaire, so that their individual impressions can be captured prior to the collective discussion, minimising bias.

- A semiscripted debriefing session will follow soliciting feedback from all participants. The audio/ video recording will continue until the end of the debrief.

8. Following each scenario, the simulation team will restock any disposables and ensure the trauma bay has been restored to 'ready' status for any ensuing trauma patients. A similar process will be enacted should a real trauma patient require care while the simulation is in process.

\section{Outcome measures}

The primary outcome measures of the study will be a list of LST codes identified through video review, including their frequency and prioritisation. Secondary outcome measures will be a list of LST codes identified through participant debriefings and postsimulation questionnaire, including their frequency and prioritisation.

\section{Data analysis}

A modified FA to identify LSTs

$\mathrm{FA}^{44}$ will be used to analyse video recordings of each simulation. FA shares similarities with thematic analyses by producing descriptions of data that are clustered into themes. In our study, we will identify LSTs and categorise them by theme.
The general process for implementing the FA is summarised below, closely following the steps outlined by Gale et al. ${ }^{44}$

1. One HF observer will transcribe key events in the video (eg, at $1 \mathrm{~min} 26 \mathrm{~s}$ of the video, the general surgeon walks into room and does not put on gloves prior to providing hands-on care). A clinician will review the events for clinically relevant omissions or misinterpretations.

2. At least two HF team members will perform multiple viewings of the recordings to familiarise themselves with the various activities and processes occurring during the scenario.

3. The HF team members will independently assign an LST code(s) to each transcribed event. These codes will be developed inductively and will be a concise description of the LST observed. An LST code may be associated with multiple events (eg, 'ultrasound machine turns off by itself' and 'glidescope screen remains blank when turned on' may have the same code of 'equipment malfunction'). In turn, LST codes will be grouped with other relevant codes using the HF elements previously described (individual skills/knowledge and team interaction; tools/ technology, task, physical environment and layout; and organisation characteristics). For example, the LST code, 'equipment malfunction', could be grouped with 'poor device usability', under the tools/technology theme.

4. HF raters will review their collective codes for overlap (eg, both raters may have identified the same LST in principle, but need to come to consensus on the wording moving forward) and discuss whether the themes are appropriate to capture the LSTs identified. If the a priori themes are not sufficient, they may be modified or discarded in favour of new themes. This set of themes and codes will comprise the 'analytical framework'.

5. The analytical framework will be used by HF raters to independently code subsequent simulations, but raters will retain the ability to add new codes if the observed LSTs are not felt to be represented in the latest version of the analytical framework. The HF reviewers will meet to resolve discrepancies between how they applied the codes and come to consensus for how codes should be applied and what codes should be in the analytical framework. The analytical framework will then be updated and used to code a subsequent simulation.

6. When the HF raters have achieved relative stability for code assignment, an inter-rater reliability (IRR) test will be performed on a simulation that they have independently coded but not yet discussed. If an acceptable IRR (ie, $\kappa>0.7$ ) is not achieved, raters will come to consensus on an updated coding framework, and repeat the process for the next analysed scenario until IRR $>0.7$ is achieved. 
7. Once acceptable IRR is established, a single HF rater will proceed to code all subsequent simulations (and retrospectively update previously coded simulations to ensure the analytical framework is applied uniformly to all data).

8. All coded simulations will be charted into the framework matrix for review by the study team.

A key output of FA is the development of a matrix, which will be finalised in step 8 . This matrix will be a table that organises the codes by the simulation in which they occur, and by the LST theme (figure 3). In our study, each row will represent 1 of the 12 simulations conducted, and each column will represent one of the LST themes. The intersection of a row and column will contain all codes that occurred during a scenario related to the corresponding LST theme in that row (figure 3). Therefore, the matrix will concisely summarise the LSTs within and across simulations, and will facilitate analysis of their frequency and typology with helpful context.

The purpose of conducting an IRR analysis (step 6) will be to determine if the analytical framework has become robust enough that two different raters can reliably categorise an identified LST into the themes in the analytical framework. If an acceptable IRR is achieved, a single rater will be sufficient to assign the codes in subsequent simulations. Should subsequent simulations require adjustments to LST themes, the IRR process will be restarted and once achieved, and the new framework will be applied to previously coded simulations for consistency.

\section{Prioritisation of LSTS}

Once the framework matrix is finalised, the study team will prioritise LST codes where interventions are needed. The criteria for prioritisation will be based on the Healthcare Failure Mode and Effect Analysis (HFMEA) decision tree, in which a 'hazard score' is first calculated for each LST code by multiplying the frequency of its occurrence and the severity of its effect on the patient. ${ }^{50}$ Members of the study team with clinical experience in trauma resuscitation will rate frequency and severity of each LST code on a four-point scale, devising an approximate criteria as a guide (eg, frequency of 4 refers to an LST that happens twice or more during every trauma case, frequency of 1 refers to LST that occurs once in a year or less).

LSTs scoring higher than a specific threshold determined by the study team (eg, a hazard score of 9 or higher), or LSTs that could result in total system failure by themselves, will then be further filtered by assessing whether (1) control measure(s) are already in place (ie, technology or processes already in place that are likely to identify the LST) and/or (2) the LSTs are likely to become so obvious that healthcare providers will discover them before they can lead to error(s) (ie,
Figure 3 Example of FA matrix output. This figure is for illustration purposes only (only 2 out of the 12 simulations are pictured, and the number and name of the themes will depend on the data). The intersection of rows and columns will summarise the LSTs identified in each simulation by either (1) a priori themes derived from HF system elements or (2) themes developed inductively over the course of the coding process.

\begin{tabular}{|c|c|c|c|}
\hline & $\begin{array}{l}\text { Theme 1: } \\
\text { Tools/Technology }\end{array}$ & $\begin{array}{l}\text { Theme 2: Physical } \\
\text { Environment and } \\
\text { Layout }\end{array}$ & Theme 3:... \\
\hline Simulation 1 & $\begin{array}{l}\text { LST Code: } \\
\text { Equipment } \\
\text { malfunction } \\
\text { Timestamp: 1:34 } \\
\text { Transcript: "Bag } \\
\text { valve mask } \\
\text { spontaneously } \\
\text { breaks apart while } \\
\text { anesthesiologist is } \\
\text { bagging patient" }\end{array}$ & $\begin{array}{l}\text { LST Code: Unknown } \\
\text { location Timestamp: } \\
5: 52 \\
\text { Transcript: "Team } \\
\text { leader asks for surgical } \\
\text { airway tray, but nurse is } \\
\text { unable to find it at the } \\
\text { equipment shelves" }\end{array}$ & - \\
\hline Simulation 2 & - & $\begin{array}{l}\text { LST Code: Team } \\
\text { member in the way } \\
\text { Timestamp: } 6: 03 \\
\text { Transcript: "TTL asks } \\
\text { for chest X-ray but x-ray } \\
\text { technician cannot get into } \\
\text { position because nurse is } \\
\text { finishing IV blood draws" }\end{array}$ & - \\
\hline
\end{tabular}


detectability). Team members with clinical experience in trauma resuscitation will come to consensus on whether the answers to these two questions are yes or no. As a result, the output will prioritise LSTs by their hazard score and lack of mitigation in the current system.

\section{SAFETY AND ETHICAL CONSIDERATIONS Participants and consent}

This study has institutional research ethics board approval (SMH REB \#15-046). All residents and staff who participate as trauma team members will receive a description of the study via email. Consent will be established in advance of study participation either during hospital orientation for residents or through dedicated information sessions for staff.

The risks of participating in the study are no higher than when providing routine care to patients because:

- all study findings will be de-identified,

- all study findings will be presented in aggregate fashion (with the exception of immediate safety concerns (see the Dissemination strategy section),

- no real patient care will be administered.

Disruptions to actual patient care during the simulations are no greater than those present when real trauma cases disrupt typical operations. In cases where a real trauma patient is expected, the study will immediately be cancelled/stopped and the simulation team will withdraw and restore the trauma bay to its 'ready' state.

\section{Standardised patient}

For scenarios that involve SP, we will carefully design the case such that invasive or surgical procedures are not clinically indicated. The SP will also be thoroughly briefed to avoid any actions or wording that might trigger invasive care procedures. In addition, confederate team members will be present to ensure SP safety.

\section{Early dissemination of important safety findings}

In the event that critical safety issues are discovered that present severe harm to patients as determined by either clinical or HF team members, the results will be shared immediately with the trauma programme leadership and the appropriate clinical members.

To preserve participant anonymity should such an event occur, all communications regarding a critical safety issue will be anonymised and focus on system improvements (eg, process and environmental considerations) rather than the study participants.

\section{DISSEIMINATION STRATEGY}

We will disseminate study results regardless of the findings. This study will provide insights into current practice and the incidence of LSTs in an established trauma centre. Study results will be of interest to a variety of key stakeholders, given the interprofessional nature of our study.
During the study, we will share an interim report of key LSTs identified to date at our institution's trauma rounds. On study completion, results will be presented at scientific meetings and published in peer-reviewed medical journals.

\section{LIMITATIONS}

\section{Limitations of ISS}

ISS is new to our trauma centre. To increase buy-in and engagement from hospital administration and potential participants, we will purposely schedule ISS during times of lower patient volumes to reduce the impact on actual clinical operations. ${ }^{51}$ As a result, we may fail to capture some of the issues related to trauma cases occurring during peak patient volumes.

In addition, there is a risk that some staff may be resistant to participation in a simulation as it prevents them from providing real patient care, and some staff may even opt to return to their usual duties on recognising the trauma activation was for simulation purposes. To counter this, we will provide regular updates to hospital administration and hospital staff to ensure that everyone is aware of the study and its goals.

The usual limitations of simulation exist in our study. Participants may not behave in the same manner they would during an actual trauma resuscitation. Simulation requires participants to 'suspend disbelief' to maximise the learning opportunities. Challenges related to simulation authenticity are tempered when sessions occur in situ. ${ }^{52}$ By developing and conducting realistic scenarios with the same equipment and processes available as actual trauma resuscitations, we expect to improve participant engagement and buy-in.

The usual limitations apply regarding the Hawthorne effect-the notion that participants in research studies may modify their behaviour in response to being studied ${ }^{53}$ While it is impossible to guarantee that clinicians' behaviour remains unchanged as a result of being observed, our participants will be familiar with observers in the trauma bay, a common occurrence at an academic trauma centre. Although there is some debate on the impact of the Hawthorne effect on human behaviour, ${ }^{54}$ observers will remain as unobtrusive as possible to minimise the impact on participant behaviour. We expect that any behaviour change resulting from the Hawthorne effect is only likely to decrease the number of errors due to increased vigilance of staff while being observed. Thus, errors discovered under these circumstances can reasonably be considered a best-case outcome.

Given these scenarios are unannounced and participants are not briefed and oriented in the usual manner for centre-based simulation, confederate actors are needed to aid participants and ensure appropriate scenario flow. This may inadvertently provide additional advantages to the trauma team in terms of resourcing, but our confederate team members will be careful to perform routine tasks and/or requested tasks rather 
than initiate important scenario altering actions on their own.

Finally, other interventions (eg, internal quality improvement processes at our hospital) unrelated to our study may be introduced into the trauma bay, which could impact our results. Since our study is designed to capture the realities of our trauma resuscitation programme, we see no need to attempt to control for these changes. Indeed, our study may help assess what type of impact these changes have produced.

\section{Limitations of data analysis approach}

As a pilot project, we are limiting our study to 12 simulations. This means that the objective of the study is exploratory, and not intended to establish statistical significance for changes to LSTs over time, or between trauma teams. We restricted our study to four different scenarios, so that each scenario could be repeated and allow us to monitor variations in how they unfold. We recognise that our findings may not be wholly representative of the true frequency, variety or potential severity of LSTs that can occur in trauma resuscitation-this is not our goal. This study's unique contribution is that it provides an indepth and rigorous video review of ISS in trauma resuscitation in order to provide greater contextual detail on a subset of potential LSTs, rather than identify all possible LSTs.

The number of simulations is also limited because we anticipate that FA will be extremely time-consuming. Unlike most instances where FA has been used, we face the unwieldy process of transcribing a video (as opposed to a verbal interview), which adds significant time investments because we must assess which events in the video are worthy of inclusion in the transcription. To counter the risk of bias, and to minimise the extensive time requirements on clinical team members, the HF team members will perform the (1) transcription and (2) coding of each simulation, and seek a review by at least one clinical team member at the completion of each step.

Given the specificity of ISS to the LSTs in our specific trauma centre, our findings may not be applicable to other trauma centres. While we anticipate that there may be some idiosyncratic LSTs unique to our centre, the general themes of LSTs we identify and the methodology we have outlined here should hold value and transferability to those seeking to repeat this approach in their local context. These answers will provide meaningful contributions to advancing the literature, and help justify the intense resource investment.

\footnotetext{
Author affiliations

${ }^{1}$ Research and Innovation, North York General Hospital, Toronto, Ontario, Canada

${ }^{2}$ HumanEra, University Health Network, Toronto, Ontario, Canada

${ }^{3}$ Department of Emergency Medicine, St Michael's Hospital, Toronto, Ontario, Canada

${ }^{4}$ Division of Emergency Medicine, Department of Medicine, University of Toronto, Toronto, Ontario, Canada
}

${ }^{5}$ Allan Waters Family Simulation Centre, St Michael's Hospital, Toronto, Ontario, Canada

${ }^{6}$ Human Factors and Usability Laboratory, Universidade Federal de Itajubá, Itajubá, Brazil

${ }^{7}$ Department of Pediatrics, University of Toronto, Toronto, Ontario, Canada ${ }^{8}$ Institute of Health Policy, Management and Evaluation, University of Toronto, Toronto, Ontario, Canada

Acknowledgements The authors wish to thank Amanda McFarlan for her assistance in the trauma registry review, and Sandro Rizoli, Najma Ahmed Glen Bandiera and Doug Sinclair for their support and mentorship. The authors also wish to thank Nazanin Khodadoust, Susan Zelko, Ashley Rosen and Roger Chow for their contributions to simulation design and logistical and planning support.

Contributors AP and $\mathrm{CH}$ designed and led the study. AP, $\mathrm{CH}$ and $\mathrm{KW}$ led the postscenario debriefs. MF, SP, APSSA and PT performed the framework analysis and inter-rater reliability analysis. AP, $\mathrm{CH}, \mathrm{KW}, \mathrm{AG}, \mathrm{MM}$, and $\mathrm{DC}$ validated the scenarios and helped execute the simulations. MF, AP and PT wrote the paper. All authors critically reviewed and edited the paper.

Funding This work was supported by grants from the Royal College of Physicians and Surgeons (Medical Education Research Grant), SIM-one/ IDEAS/CPSI Simulation for Safety and Quality Improvement Program and St Michael's Hospital AFP Innovation Fund.

Disclaimer The funding sources had no role in the design of this study and will not have any role in the study's implementation, data analysis or dissemination of study results.

\section{Competing interests None declared}

Ethics approval St. Michael's Hospital, Toronto, Ontario, Canada.

Provenance and peer review Not commissioned; externally peer reviewed.

Data sharing statement As our manuscript is for a study protocol, and not a completed research study, we have no additional data sets to share at this time.

Open Access This is an Open Access article distributed in accordance with the Creative Commons Attribution Non Commercial (CC BY-NC 4.0) license, which permits others to distribute, remix, adapt, build upon this work noncommercially, and license their derivative works on different terms, provided the original work is properly cited and the use is non-commercial. See: http:// creativecommons.org/licenses/by-nc/4.0/

\section{REFERENCES}

1. Chua WC, D'Amours SK, Sugrue M, et al. Performance and consistency of care in admitted trauma patients: our next great opportunity in trauma care? ANZ J Surg 2009;79:443-8.

2. Clarke JR, Spejewski B, Gertner AS, et al. An objective analysis of process errors in trauma resuscitations. Acad Emerg Med 2000;7:1303-10.

3. Teixeira PGR, Inaba K, Hadjizacharia $\mathrm{P}$, et al. Preventable or potentially preventable mortality at a mature trauma center. J Trauma 2007;63:1338-46. discussion 1346-7.

4. Sugrue M, Caldwell E, D'Amours S, et al. Time for a change in injury and trauma care delivery: a trauma death review analysis. ANZ J Surg 2008;78:949-54.

5. Brennan TA, Leape LL, Laird NM, et al. Incidence of adverse events and negligence in hospitalized patients. Results of the Harvard Medical Practice Study I. N Engl J Med 1991;324:370-6.

6. Pucher $\mathrm{PH}$, Aggarwal R, Twaij A, et al. Identifying and addressing preventable process errors in trauma care. World J Surg 2013;37: 752-8.

7. Gruen RL, Jurkovich GJ, Mclntyre LK, et al. Patterns of errors contributing to trauma mortality: lessons learned from 2,594 deaths. Ann Surg 2006;244:371-80.

8. Georgiou A, Lockey DJ. The performance and assessment of hospital trauma teams. Scand J Trauma Resusc Emerg Med 2010;18:66.

9. Patterson MD, Geis GL, Falcone RA, et al. In situ simulation: detection of safety threats and teamwork training in a high risk emergency department. BMJ Qual Saf 2013;22:468-77. 
10. Pronovost PJ, Goeschel CA, Marsteller JA, et al. Framework for patient safety research and improvement. Circulation 2009;119:330-7.

11. Fitzgerald $\mathrm{M}$, Gocentas R, Dziukas $\mathrm{L}$, et al. Using video audit to improve trauma resuscitation-time for a new approach. Can J Surg 2006;49:208-11.

12. Carayon P, Xie A, Kianfar S. Human factors and ergonomics as a patient safety practice. BMJ Qual Saf 2014;23:196-205.

13. International Ergonomics Association. What is ergonomics? [Internet]. Definition and domains of ergonomics. 2000. (cited 18 May 2016). http://www.iea.cc/whats/index.html

14. Holden RJ, Carayon P, Gurses AP, et al. SEIPS 2.0: a human factors framework for studying and improving the work of healthcare professionals and patients. Ergonomics 2013;56:1669-86.

15. Reason J. Human error: models and management. $B M J$ 2000;320:768-70.

16. Reason J. A systems approach to organizational error. Ergonomics 1995;38:1708-21.

17. Vicente K. The human factor: revolutionizing the way we live with technology. Canada: Vintage, 2010.

18. Rasmussen J. Risk management in a dynamic society: a modelling problem. Saf Sci 1997;27:183-213.

19. Vincent $\mathrm{C}$. Understanding and responding to adverse events. N Engl $J$ Med 2003;348:1051-6.

20. Vincent C, Taylor-Adams S, Chapman EJ, et al. How to investigate and analyse clinical incidents: clinical risk unit and association of litigation and risk management protocol. BMJ 2000;320:777-81.

21. Riley W, Davis S, Miller KM, et al. Detecting breaches in defensive barriers using in situ simulation for obstetric emergencies. Qual Saf Health Care 2010;19(Suppl 3):i53-6.

22. Wetzel EA, Lang TR, Pendergrass TL, et al. Identification of latent safety threats using high-fidelity simulation-based training with multidisciplinary neonatology teams. Jt Comm J Qual Patient Saf 2013;39:268-73.

23. Geis GL, Pio B, Pendergrass TL, et al. Simulation to assess the safety of new healthcare teams and new facilities. Simul Healthc 2011;6:125-33.

24. Guise J-M, Mladenovic J. In situ simulation: identification of systems issues. Semin Perinatol 2013;37:161-5.

25. Blike GT, Christoffersen K, Cravero JP, et al. A method for measuring system safety and latent errors associated with pediatric procedural sedation. Anesth Analg 2005;101:48-58. table of contents.

26. Wheeler DS, Geis G, Mack EH, et al. High-reliability emergency response teams in the hospital: improving quality and safety using in situ simulation training. BMJ Qual Saf 2013;22:507-14.

27. O'Leary F, McGarvey K, Christoff $A$, et al. Identifying incidents of suboptimal care during paediatric emergencies-an observational study utilising in situ and simulation centre scenarios. Resuscitation 2014;85:431-6.

28. Hunt EA, Hohenhaus SM, Luo X, et al. Simulation of pediatric trauma stabilization in 35 North Carolina emergency departments: identification of targets for performance improvement. Pediatrics 2006;117(3):641-8.

29. Lighthall GK, Poon T, Harrison TK. Using in situ simulation to improve in-hospital cardiopulmonary resuscitation. Jt Comm J Qual Patient Saf 2010;36:209-16.

30. Patterson MD, Blike GT, Nadkarni VM. In situ simulation: challenges and results. In: Henriksen K, Battles JB, Keyes MA, et al. eds. Advances in patient safety: new directions and alternative approaches (Vol 3: Performance and Tools). Rockville, MD: Agency for Healthcare Research and Quality (US), 2011:1-18.

31. Kobayashi L, Dunbar-Viveiros JA, Devine J, et al. Pilot-phase findings from high-fidelity in situ medical simulation investigation of emergency department procedural sedation. Simul Healthc 2012;7:81-94.

32. Kobayashi L, Dunbar-Viveiros JA, Sheahan BA, et al. In situ simulation comparing in-hospital first responder sudden cardiac arrest resuscitation using semiautomated defibrillators and automated external defibrillators. Simul Healthc 2010;5:82-90.

33. Burton KS, Pendergrass TL, Byczkowski TL, et al. Impact of simulation-based extracorporeal membrane oxygenation training in the simulation laboratory and clinical environment. Simul Healthc 2011;6:284-91.

34. Calhoun AW, Boone MC, Peterson EB, et al. Integrated in-situ simulation using redirected faculty educational time to minimize costs: a feasibility study. Simul Healthc 2011;6:337-44.

35. Weinstock PH, Kappus LJ, Garden A, et al. Simulation at the point of care: reduced-cost, in situ training via a mobile cart. Pediatr Crit Care Med 2009;10:176-81

36. Oakley E, Stocker S, Staubli G, et al. Using video recording to identify management errors in pediatric trauma resuscitation. Pediatrics 2006;117:658-64.

37. Holcomb JB, Dumire RD, Crommett JW, et al. Evaluation of trauma team performance using an advanced human patient simulator for resuscitation training. J Trauma 2002;52:1078-85. discussion 10856.

38. Brooks AJ, Phipson M, Potgieter A, et al. Education of the trauma team: video evaluation of the compliance with universal barrier precautions in resuscitation. Eur J Surg 1999;165:1125-8.

39. Ritchie PD, Cameron PA. An evaluation of trauma team leader performance by video recording. Aust N Z J Surg 1999;69:183-6.

40. Townsend RN, Clark R, Ramenofsky ML, et al. ATLS-based videotape trauma resuscitation review: education and outcome. $J$ Trauma 1993;34:133-8.

41. Bergs EAG, Rutten FLPA, Tadros $\mathrm{T}$, et al. Communication during trauma resuscitation: do we know what is happening? Injury 2005;36:905-11.

42. Mackenzie CF, Jefferies NJ, Hunter WA, et alComparison of self-reporting of deficiencies in airway management with video analyses of actual performance. LOTAS Group. Level one trauma anesthesia simulation. Hum Factors 1996;38:623-35.

43. Mackenzie CF, Xiao Y, Hu F-M, et al. Video as a tool for improving tracheal intubation tasks for emergency medical and trauma care. Ann Emerg Med 2007;50:436-42, 442.e1.

44. Gale NK, Heath G, Cameron E, et al. Using the framework method for the analysis of qualitative data in multi-disciplinary health research. BMC Med Res Methodol 2013;13:117

45. Dixon-Woods M. Using framework-based synthesis for conducting reviews of qualitative studies. BMC Med 2011:9:39.

46. Ward DJ, Furber C, Tierney $\mathrm{S}$, et al. Using framework analysis in nursing research: a worked example. J Adv Nurs 2013;69:2423-31.

47. Chiniara G, Cole G, Brisbin K, et al. Simulation in healthcare: a taxonomy and a conceptual framework for instructional design and media selection. Med Teach 2013;35:e1380-95.

48. Hamstra SJ, Brydges R, Hatala R, et al. Reconsidering fidelity in simulation-based training. Acad Med 2014;89:387-92.

49. Surcouf JW, Chauvin SW, Ferry J, et al. Enhancing residents' neonatal resuscitation competency through unannounced simulation-based training. Med Educ Online 2013;18:1-7.

50. DeRosier J, Stalhandske E, Bagian JP, et al. Using health care failure mode and effect analysis ${ }^{T M}$ : the VA National Center for patient safety's prospective risk analysis system. Jt Comm J Qual Improv 2002;28:248-67.

51. Ruddy RM, Patterson MD. Medical simulation: a tool for recognition of and response to risk. Pediatr Radiol 2008;38(Suppl 4):S700-6.

52. Sørensen JL, van der Vleuten C, Rosthøj S, et al. Simulation-based multiprofessional obstetric anaesthesia training conducted in situ versus off-site leads to similar individual and team outcomes: a randomised educational trial. BMJ Open 2015;5:e008344.

53. Parsons HM. What happened at Hawthorne?: new evidence suggests the Hawthorne effect resulted from operant reinforcement contingencies. Science 1974;183:922-32.

54. Adair JG. The Hawthorne effect: a reconsideration of the methodological artifact. J Appl Psychol 1984;69:334. 